POS $\quad$ PROCEEDINGS

\title{
Heavy-light current-current correlators
}

\section{Jonna Koponen*, Christine T. H. Davies, for HPQCD}

University of Glasgow, UK

E-mail: j.koponen@physics.gla.ac.uk

\section{Kent Hornbostel}

Southern Methodist University, Dallas, Texas, USA

\section{Eduardo Follana}

Universidad de Zaragoza, Spain

\section{G. Peter Lepage}

Cornell University, Ithaca, New York, USA

\section{Craig McNeile}

Bergische Universität Wuppertal, Germany

\section{Junko Shigemitsu}

The Ohio State University, Columbus, Ohio, USA

\section{Matthias Steinhauser}

Karlsruhe Institute of Technology, Germany

The current-current correlator method has been used successfully to obtain very accurate results for quark masses and the coupling $\alpha_{s}$. The calculations were done using Highly Improved Staggered Quarks (HISQ) and heavy-heavy meson correlators. We now extend this work to the significantly more challenging heavy-light case, reporting the first results here. The aim is to determine nonperturbative $Z$ factors for NRQCD heavy-light currents, but first we test the method in the HISQ case where $Z=1$.

The XXVIII International Symposium on Lattice Filed Theory

June 14-19,2010

Villasimius, Sardinia Italy

\footnotetext{
*Speaker.
} 


\section{Motivation}

In the study of semileptonic and leptonic processes, like $B \rightarrow \pi l v$ and $B \rightarrow l v$, the nonperturbative $Z$ factors for heavy-light currents are needed. One way to try to calculate these (nonperturbatively on the lattice) is the current-current correlator method, that has been successfully tested and used in the heavyonium case [1,2]. We now want to extend these results and use the same method in the heavy-light case. Here we report on the first results using the HISQ action. The eventual aim is to extract NRQCD heavy-light $Z$ factors.

\section{Current-current correlator method}

The idea is to match time moments of meson correlators to energy-derivative moments at $q^{2}=0$ of polarization functions $\Pi$ calculated in continuum QCD perturbation theory to high order.

The pseudoscalar current-current correlators are defined as

$$
G(t)=a^{6} \sum_{\vec{x}}\left(a m_{q}\right)^{2}\left\langle 0\left|j_{5}(\vec{x}, t) j_{5}(0,0)\right| 0\right\rangle .
$$

Then the time moments are

$$
G_{n}=\sum_{t}\left(\frac{t}{a}\right)^{n} G(t)
$$

(see e.g. $[1,2]$ ). To help reducing the errors we divide each moment by the tree level value, $G_{n}^{(0)}$, and define reduced moments $R_{n}$ as

$$
R_{4}^{\text {latt }}=\frac{G_{4}}{G_{4}^{(0)}}, \quad \text { and } \quad R_{n}^{\text {latt }}=\left(\frac{G_{n}}{G_{n}^{(0)}}\right)^{\frac{1}{n-4}} \quad \text { for } \quad n \geq 6 .
$$

In the continuum the reduced moments are

$$
R_{4}^{\text {cont }}=\frac{g_{4}}{g_{4}^{(0)}}, \quad \text { and } \quad R_{n}^{\text {cont }}=\frac{m_{\eta_{h}}}{2 m_{h}(\mu)} \frac{g_{n}}{g_{n}^{(0)}} \quad \text { for } \quad n \geq 6 .
$$

The $g_{n}$ are perturbative series in $\alpha_{s}(\mu)$, known for the heavy-heavy case through $\alpha_{s}^{3}(\mu)$ [3] and for heavy-light through $\alpha_{s}^{2}(\mu)$ [4]. The mass $m_{h}$ is the heavy quark mass in the $\overline{\mathrm{MS}}$ scheme at the scale $\mu$. Comparing the lattice and continuum $R_{n}$ allows us to extract the mass ratio $m_{\eta_{h}} /\left(2 m_{h}(\mu)\right)$, and thus the quark mass. The calculation above is for the case with no $Z$ factor. If the lattice current has a $Z$ factor then that can also be extracted.

\section{Heavy-light JJ correlators}

We compare lattice calculations to continuum perturbation theory through $\alpha_{s}^{2}(\mu)$. In this work we have used coarse, fine, superfine and ultrafine MILC lattice configurations. We calculate heavy-light correlators using the HISQ action [5] for both quarks with several heavy quark masses from charm up to the $b$ quark mass. Note that $Z=1$ in the HISQ case. Some of the calculated reduced moments $R_{n}$ are shown in Fig. 1 as examples of our results. Comparing heavy-strange, heavy-charm and heavy-heavy correlator reduced moments shows that we can clearly distinguish between these three cases. In the following subsections we address some challenges of the heavylight calculations. 

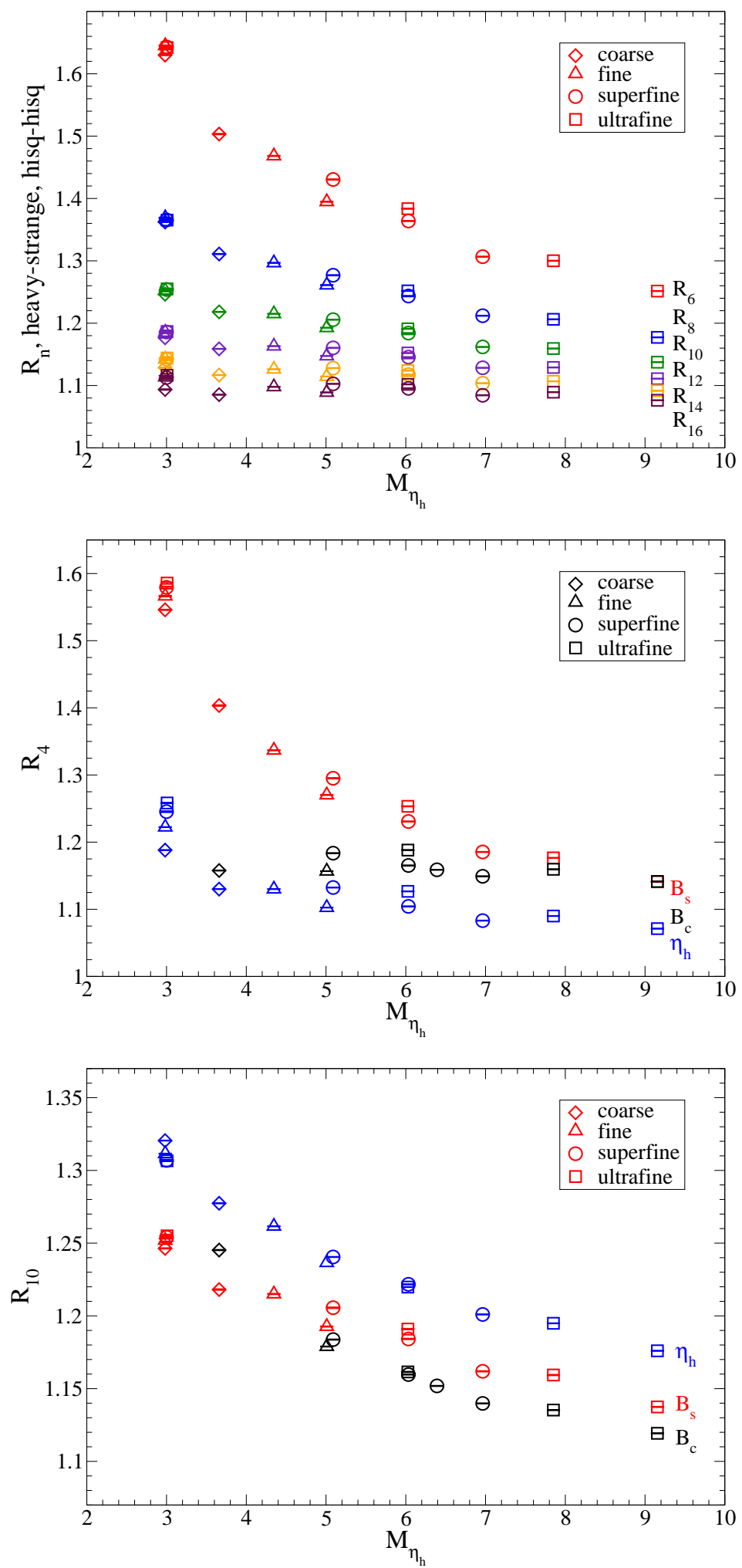

Figure 1: Top figure: Heavy-strange correlator reduced moments $R_{n}$ as a function of heavy-heavy meson mass $M_{\eta_{h}}$ (in GeV). The other two figures show the heavy-strange, heavy-charm and heavy-heavy reduced moments $R_{4}$ and $R_{10}$ as a function of $M_{\eta_{h}}$. The range is from charm (at about $3 \mathrm{GeV}$ ) to $b$ (about $10 \mathrm{GeV}$ ). 


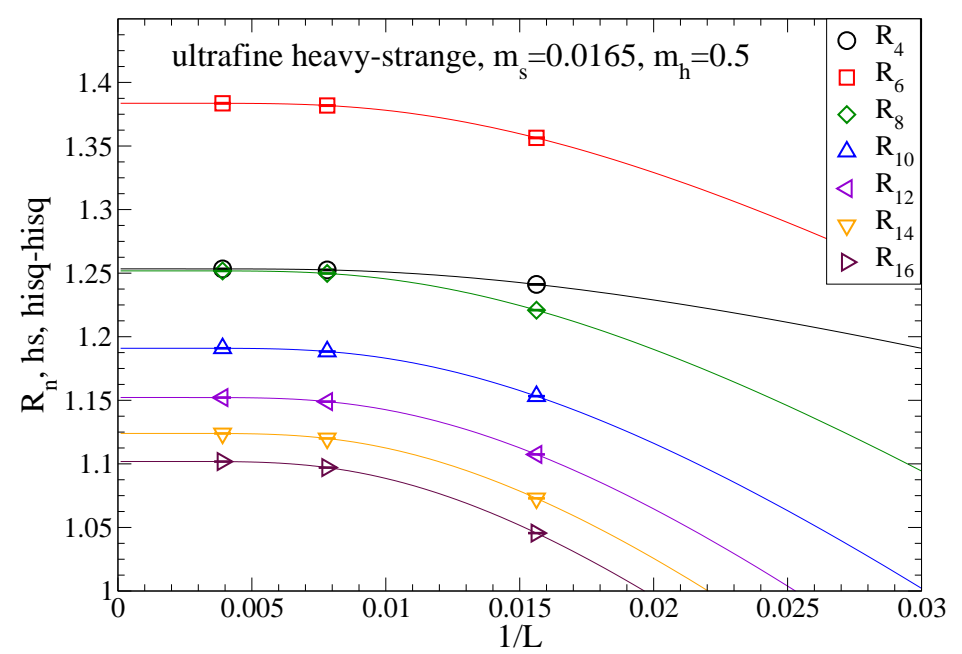

Figure 2: The reduced moments in the free, non-interacting theory depend on the volume.

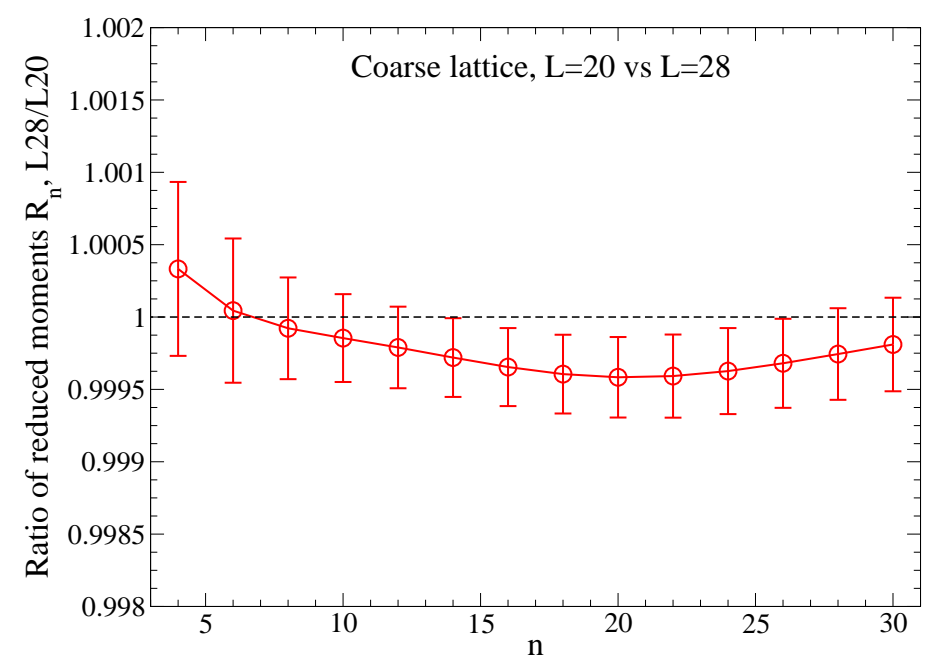

Figure 3: The ratio of reduced moments calculated on two different coarse lattices (one with $L=20$, other one with $L=28$ ) shows that there is no volume dependence in the interacting case.

\subsection{Volume dependence}

The tree level (free) moments depend on volume - note that this is an artifact of the free case only. This is illustrated in Figures 2 and $3-$ the $R_{n}$ depend on volume in the non-interacting theory, but not in the interacting case. Therefore we need to calculate the tree level moments in the infinite volume limit. We do this by calculating the free moments using different volumes, $L^{3}$, and fitting them with

$$
A_{0}+A_{1} \frac{e^{-A_{2} L}}{L}
$$

The result in the infinite volume limit is then simply given by the fit parameter $A_{0}$. 


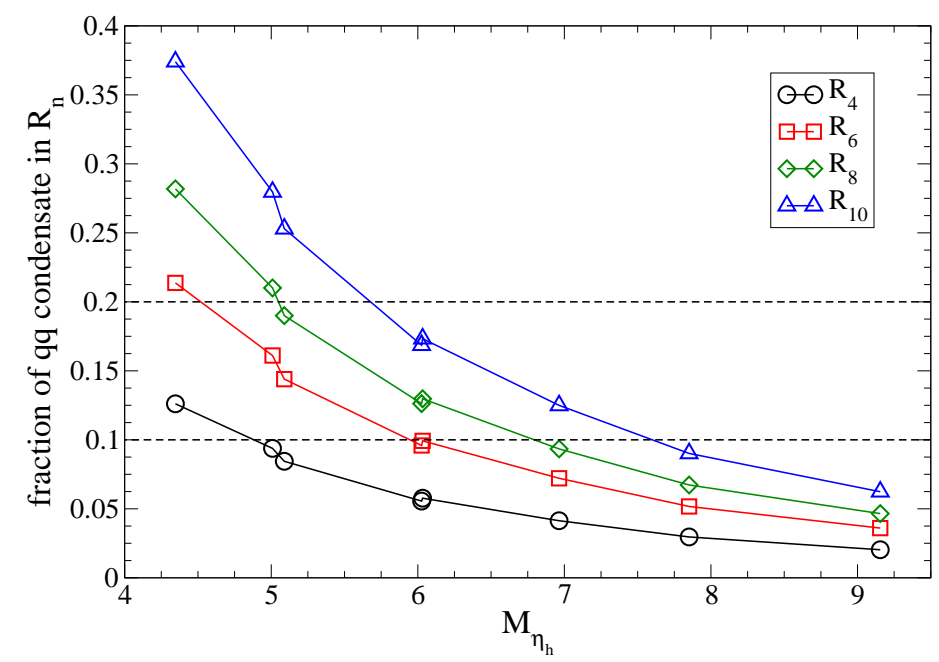

Figure 4: Fraction of the tree level $q \bar{q}$ condensate in reduced moment $R_{n}$ as a function of the heavy-heavy meson mass $M_{\eta_{h}}$ (in GeV).

\subsection{Quark condensate}

The quark condensate appears in the reduced moment $R_{n}$ at tree level as [6]

$$
\frac{4 \pi^{2}}{3} \frac{(n-1)(n-2)(n-3)\left[-\frac{m_{h}}{m_{l}}+\frac{n}{2}\right]}{1+\frac{(n-3) m_{l}}{m_{h}}} \frac{\left\langle m_{l} \psi \bar{\psi}\right\rangle}{m_{h}^{4}} .
$$

The quark condensate is not present in the heavy-heavy case, but it is sizeable in the heavy-light case - the fraction of tree level $q \bar{q}$ condensate in $R_{n}$ can easily be $10-30 \%$ for heavy quark masses masses between $c$ and $b$, as can be seen in Fig. 4. Note that the leading term is $1 / m_{h}^{3}$. This poses a challenge, as the $\alpha_{s}$ corrections to the condensate are not known. The gluon condensate contribution is much smaller and can be safely neglected in the analysis.

\section{3 $m_{l} / m_{h}$ corrections to perturbative series}

Perturbation theory with $m_{q}=0$ is not sufficient, as $m_{l}(\mu) / m_{h}(\mu)$ corrections become important for $B_{c}: m_{c}(\mu) / m_{b}(\mu) \approx 0.22$. At small values of the ratio the $m_{l}(\mu) / m_{h}(\mu)$ expansion is good, i.e. it works for $B_{s}$. At large values of the ratio the expansion is not good enough. This is illustrated in Fig. 5. However, we now have the exact coefficients for given ratios $m_{l}(\mu) / m_{h}(\mu)$ for tree level (shown in the plot as bursts) and order $\alpha_{s}$, so this problem can be partly avoided. The exact coefficients are still needed for $\alpha_{s}^{2}$ and higher orders.

\subsection{Fits}

We fit the lattice data $R_{n}^{\text {latt }}, n \geq 6$, with

$$
\begin{aligned}
R_{n}^{\mathrm{fit}}= & \left(\frac{m_{\eta_{h}}}{2 m_{h}(\mu)}\right)\left(1+c_{1} \alpha_{s}+c_{2} \alpha_{s}^{2}+c_{3} \alpha_{s}^{3}+c_{4} \alpha_{s}^{4}+c_{5} \alpha_{s}^{5}+c_{6} \alpha_{s}^{6}+q \bar{q} \quad \text { condensate }\right) \\
& \left(1+b_{1}\left(a m_{h}(\mu)\right)^{2}+b_{2}\left(a m_{h}(\mu)\right)^{4}+b_{3}\left(a m_{h}(\mu)\right)^{6}+d_{1} a^{2}+d_{2} a^{4}\right)
\end{aligned}
$$



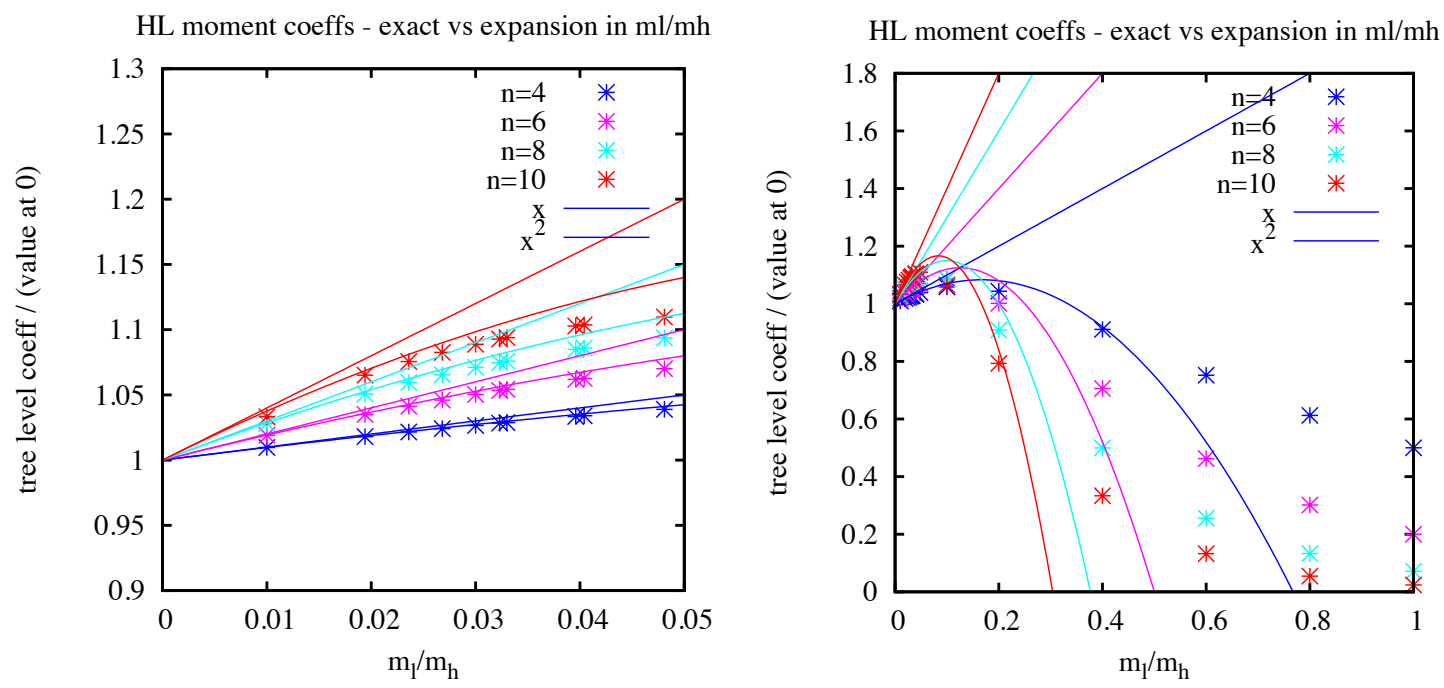

Figure 5: $m_{l}(\mu) / m_{h}(\mu)$ corrections to continuum perturbation theory coefficients. Here $x=m_{l} / m_{h}$. The tree level values of the coefficients have been divided by the tree level value at $m_{l} / m_{h}=0$.

and choose the scale $\mu=m_{h}$. We take the first few coefficients ( $c_{1}$ and $c_{2}$ in the heavy-light case) from continuum perturbation theory, and treat the coefficients for the higher order $\alpha_{s}$ terms as fit parameters. The quark condensate is given in Eq. 3.2 at tree level. We take the $q \bar{q}$ condensate value to be $\left\langle m_{s} s \bar{s}\right\rangle=(0.2 \mathrm{GeV})^{4}$ from the Gell-Mann - Oakes - Renner relation, allowing the $s \bar{s}$ condensate to be 0.7 times the light quark condensate. We also allow for the presence of higher order condensate terms estimating them with powers of the leading condensate. In $B_{c}$ there is no condensate contribution, and we get a good fit using the exact coefficients (order $\alpha_{s}$ ). As the lattice calculations are done at a non-zero lattice spacing $a$, we include $a$-dependent terms in the fit function - even powers of $a$ and $a m_{h}(\mu)$.

To extract the mass ratio $m_{\eta_{h}} /\left(2 m_{h}(\mu)\right)$ we use the lattice simulation data (Eq. 2.3), with $a m_{\eta_{h}} / a m_{h}$ from the lattice simulations, and compare these $R_{n}^{\text {latt }}$ to the continuum perturbation theory result (Eq. 2.4). That is, we find values for $\alpha_{\overline{\mathrm{MS}}}(\mu)$ and $m_{\eta_{h}} /\left(2 m_{h}(\mu)\right)$ that make lattice and continuum results agree for small $n>4$. This can then be combined with experimental results for the $\eta_{b}, \eta_{c}$ meson masses to obtain the quark masses. In the heavy-light case we can use the $\alpha_{\overline{\mathrm{MS}}}(\mu)$ values extracted from the heavy-heavy calculation.

To test the method in the heavy-light case we look at the mass ratio $m_{\eta_{h}} /\left(2 m_{h}(\mu)\right)$ and compare to heavy-heavy results. This is shown in Figure 6. In the heavy-strange case the fits are to one $R_{n}$ at a time, not to all $R_{n}$ simultaneously as in the heavy-heavy case. The mass ratio extracted from the heavy-strange correlator moments is the same as in the heavy-heavy case, as expected, but currently a lot less accurate.

\section{Conclusions and future}

We are extending the use of current-current correlator method, earlier used to study heavyheavy systems, to heavy-light systems. The full analysis of heavy-light data is still in progress, but we can already say that the JJ correlator method works well. The quark condensate sets some 


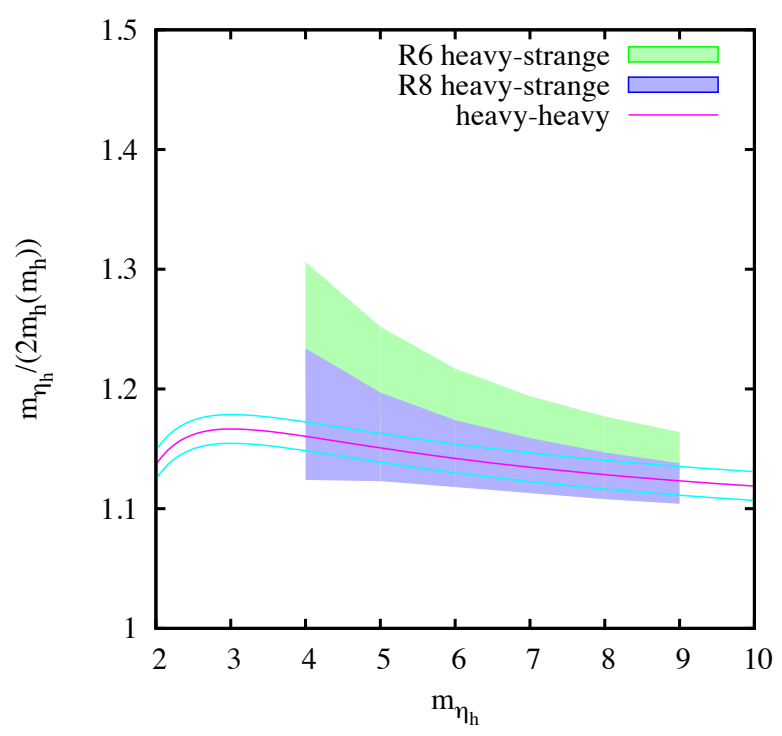

Figure 6: The mass ratio $m_{\eta_{h}} /\left(2 m_{h}(\mu)\right)$.

limitations - we can not use high moments in the $B_{s}$ case. However, in $B_{c}$ there is no condensate contribution. Our aim is to extract $Z$ for NRQCD - there the challenge is to control relativistic corrections.

\section{References}

[1] C. McNeile, C.T.H. Davies, E. Follana, K. Hornbostel, G.P. Lepage; HPQCD collaboration; Phys.Rev.D82:034512,2010, arXiv:1004.4285

[2] I. Allison, E. Dalgic, C.T.H. Davies, E. Follana, R.R. Horgan, K. Hornbostel, G.P. Lepage, C. McNeile, J. Shigemitsu, H. Trottier, R.M. Woloshyn, K.G. Chetyrkin, J.H. Kuhn, M. Steinhauser, C. Sturm; HPQCD collaboration; Phys.Rev.D78:054513,2008, arXiv:0805.2999

[3] K. G. Chetyrkin, J. H. Kuhn and C. Sturm; Eur.Phys.J. C48 (2006) 107, arXiv : hep-ph / 0604234

[4] K.G. Chetyrkin, M. Steinhauser; Eur.Phys.J. C21 (2001) 319-338, arXiv : hep-ph/ 0108017

[5] E. Follana, Q. Mason, C. Davies, K. Hornbostel, G.P. Lepage, J. Shigemitsu, H. Trottier, and K. Wong; HPQCD collaboration; Phys.Rev.D75:054502,2007, arXiv: hep-lat/ 0610092

[6] L. J. Reinders, H. R. Rubinstein and S. Yazaki; Phys. Lett. B 97 (1980) 257-260 